\title{
Physical Activity and Health-Related Quality of Life Among Community Dwelling Elderly
}

\author{
Hadeel Halaweh ${ }^{\mathrm{a}, \mathrm{c}}$, Carin Willen ${ }^{\mathrm{a}}$, Anna Grimby-Ekman ${ }^{\mathrm{b}}$, Ulla Svantesson ${ }^{\mathrm{a}}$
}

\begin{abstract}
Background: Physical activity (PA) and health-related quality of life (HRQoL) are important factors for optimal health in the elderly. Studying the association between PA and HRQoL is becoming more essential as the number of elderly people increases worldwide. This study assesses the association between PA and HRQoL among community dwelling elderly above 60 years old.
\end{abstract}

Methods: The study included 115 women and 61 men (mean age: $68.15 \pm 6.74$ years) recruited from the community and from public centers for the elderly. Data were collected using a background characteristics questionnaire (BCQ), a physical activity socio-cultural adapted questionnaire (PA-SCAQ), and the EuroQuol-5Dimensions5Levels (EQ-5D-5L) questionnaire. Between groups, comparisons were based on the PA-SCAQ by dividing the participants into three PA groups: low $(n=74)$, moderate $(n=85)$, and high $(n=17)$. Kruskal-Wallis tests were performed on the ordinal variables of the three PA groups to determine differences between the groups according to categorical variables such as gender, body mass index (BMI), and the prevalence of comorbid conditions. Mann-Whitney U tests were performed on the ordinal variables of the EuroQuol-5Dimensions (EQ-5D), and the independent sample $t$-test was performed on the EQ visual analogue scale (EQ-VAS). Spearman's rank correlation coefficient was used to examine the correlation between the EQ-5D and level of PA.

Results: Values in all dimensions of HRQoL were significantly higher $(\mathrm{P}<0.05)$ in the moderate and high PA groups compared with the low PA group. Significant correlations were recorded between the five dimensions of HRQoL and the level of PA $(\mathrm{P}<0.001)$. The low PA group showed higher prevalence of hypertension $(64 \%, \mathrm{P}<0.001)$ and diabetes $(50 \%, \mathrm{P}<0.001)$.

Manuscript accepted for publication August 26, 2015

aDepartment of Physiotherapy, Institute of Neuroscience and Physiology at Sahlgrenska Academy, University of Gothenburg, Sweden

${ }^{b}$ Health Metrics at Sahlgrenska Academy, University of Gothenburg, Sweden ${ }^{c}$ Corresponding Author: Hadeel Halaweh, Institute of Neuroscience and Physiology, Department of Physiotherapy, University of Gothenburg, Box 455, SE 40530 Goteborg, Sweden. Email: Hadeel.halaweh@neuro.gu.se

doi: http://dx.doi.org/10.14740/jocmr2307w
Conclusion: There were strong associations between higher levels of PA and all dimensions of HRQoL. Therefore, adopting a PA lifestyle may contribute to better HRQoL among community dwelling elderly above 60 years old.

Keywords: Healthy aging; Physical activity; Habitual walking; Comorbidity

\section{Introduction}

Participation in physical activity (PA) promotes healthy aging and plays an important role in improving quality of life (QoL) among elderly [1-3]. Evidence suggests that participating in regular moderate intensity PA (e.g., walking, cycling, or light sports) has significant benefits for health, including improved treatment of many diseases [4-6]. The evidence of health benefits of PA is stronger for adults 65 years old and older than for any other age group, since the consequences related to inactivity are more severe for this age group. Compared to less active elderly, active elderly have lower rates of all-cause mortality and a higher level of muscular fitness [1, 7]. Therefore, increasing PA among elderly persons has become an international priority [1]. This priority coincides with the reality that worldwide the number of elderly is increasing and the proportion of people aged over 60 years is growing faster than any other age group. This means society will need to reconsider how to care for elderly in order to maximize the health and functional capacity of elderly persons.

Aging, health status, PA, and disability all affect independence and QoL in elderly persons $[6,8,9]$. QoL is related to an individual's perception of the position in life in the context of culture and value systems and is influenced in a complex way by the person's physical health, psychological state, level of independence, and social relationships [10]. Health-related quality of life (HRQoL) is part of a multidimensional approach that considers physical, mental, and social aspects [2]. Assessing HRQoL is an essential component of healthcare evaluation [11]. Several instruments have been developed to assess HRQoL in different populations. Factors related to demographic and clinical characteristics including age, health status, culture, and language of the studied population are important determinants for using a relevant valid and 
Table 1. Clinical Characteristics of the Participants

\begin{tabular}{|c|c|c|}
\hline Clinical characteristics & Women $(n=115)$ & $\operatorname{Men}(n=61)$ \\
\hline & Mean (SD) & Mean (SD) \\
\hline Weight (kg) & $78(14.7)$ & $82(11.27)$ \\
\hline Height $(\mathrm{cm})$ & $156(0.06)$ & $170(0.06)$ \\
\hline Body mass index & $32(5.09)$ & $28(3.84)$ \\
\hline Diagnosed disease & $\mathrm{n}(\%)$ & $\mathrm{n}(\%)$ \\
\hline Yes & $101(88)$ & $51(84)$ \\
\hline No & $14(12)$ & $10(16)$ \\
\hline Cardiovascular & $22(19)$ & $17(28)$ \\
\hline Diabetes & $36(31)$ & $18(30)$ \\
\hline Hypertension & $57(50)$ & $29(48)$ \\
\hline Hyperlipidemia & $25(22)$ & $5(8)$ \\
\hline Musculoskeletal & $70(61)$ & $25(41)$ \\
\hline Osteoporosis & $28(24)$ & $2(3)$ \\
\hline \multicolumn{3}{|l|}{ Sensory function } \\
\hline Visual problems & $83(72)$ & $44(72)$ \\
\hline Hearing problems & $23(20)$ & $7(12)$ \\
\hline Taking medications & $95(83)$ & $50(82)$ \\
\hline Been hospitalized in the last year & $28(24)$ & $11(18)$ \\
\hline Regular medical checkup & $63(55)$ & $34(56)$ \\
\hline \multicolumn{3}{|l|}{ Using assistive devices } \\
\hline Glasses & $66(57)$ & $43(71)$ \\
\hline Hearing aids & $3(3)$ & $1(2)$ \\
\hline Cane & $12(10)$ & $12(20)$ \\
\hline \multicolumn{3}{|l|}{ Walks duration (min/week) } \\
\hline$<150$ & $57(50)$ & $17(28)$ \\
\hline $150-300$ & $52(45)$ & $33(54)$ \\
\hline$>300$ & $6(5)$ & $11(18)$ \\
\hline
\end{tabular}

SD: standard deviation; $\mathrm{n}$ : number of cases.

reliable HRQoL measure [11-13].

Higher levels of PA appear to be associated with better functioning and better HRQoL [2, 3, 8, 9, 14]. PA can help older adults reclaim or maintain a healthy aging process [9]. Adopting a physically active lifestyle, including increasing leisure time PA, may result in a better long term HRQoL among elderly persons $[15,16]$. Relatively regular moderate PA can help elderly prevent a decline in HRQoL and even improve their enjoyment of life [17]. Since these are important factors that promote optimal health in elderly, studying the association between PA and HRQoL is becoming more essential, especially as the number of elderly is increasing. This study assesses the association between PA and HRQoL in community dwelling elderly ( $\geq 60$ years old).

\section{Methods}

\section{Participants}

A volunteer sample of 176 community dwelling elderly Palestinians from the West Bank was recruited (115 women and 61 men; mean age: $68.15 \pm 6.74)$. Inclusion criteria consisted of women and men aged $\geq 60$ years and independence in ambulation with or without walking aids (independence in ambulation was determined based on the participant's walking capacity, if they were able to walk for at least $6 \mathrm{~m}$ independently). Exclusion criteria for the elderly included diagnosis of a severe disease that would make investigations impossible and com- 
munication deficits such as could not answer questions about age, children, and current location, time, season, and year.

In this cross-sectional study, all participants were informed about the aim of the study and signed an informed consent. The study received ethical approval from the research ethics committee of Al-Quds University, Palestine (Ref No. 1/REC/13), and the study complies with the Declaration of Helsinki.

\section{Measurements}

\section{Background characteristics questionnaire (BCQ)}

Demographic clinical descriptive data on age, gender, educational level, job status, smoking habits, medication, diagnosed disease (cardiovascular, musculoskeletal, hypertension, and others), and sensory functions (visual, hearing and speech) were registered. Anthropometric measurements (weight and height) were also recorded.

\section{Measure of activities of daily living (ADL)}

The Katz index was used to assess personal ADL [18]. The index has been described as a valid and reliable measure to determine independency level in performing ADL [19]. The assessment is based on the ability to perform an activity without assistance from another person. The Katz index of ADL includes six basic activities of daily living (BADL): bathing, dressing, toileting, transferring, continence, and feeding. The ability in performing each activity was assessed using a twopoint categorical scale: $1=$ independence and $0=$ dependence. The total score ranged from 0 (low function, dependent) to 6 (high function, independent).

\section{Measure of $P A$}

A physical activity socio-cultural adapted questionnaire (PASCAC) was used. The PA-SCAQ includes the following questions: How often do you take outdoor walks? How long are your walks? What household activities and other activities (e.g., yard work gardening) do you do? The structure of the questionnaire concerning PA domains, duration, and frequency was built on the WHO global recommendations [1] as well as by considering some culturally applicable items of the valid PA measures for elderly persons [20, 21]. Accordingly, PA variables were categorized into walking, household activities, and outdoor activities. To compute the accumulated frequency and duration of all the activities, the minutes spent per week on each of these activities were summed. The participants were categorized into three groups based on their moderate-intensity aerobic PA (MIA-PA) (walking) throughout the week: low PA (less than $150 \mathrm{~min} /$ week), moderate PA (between 150 and $300 \mathrm{~min} /$ week), and high PA (more than $300 \mathrm{~min} /$ week).

To assess whether the questionnaire's questions and the response categories were understandable by and appropriate for the participants, the PA-SCAQ was pilot tested with 10 com- munity dwelling elderly Palestinians with different levels of education (six women and four men between 62 and 83 years old). All questions and response categories were considered comprehensible, so the piloted version was not subjected to any additional modifications and was used in this study as PASCAQ.

\section{Measure of QoL: the Arabic version of EuroQuol-5Dimen-} sions-5Levels (EQ-5D-5L)

The EQ-5D-5L is a standardized, non-disease specific instrument developed for describing and valuing HRQoL. The instrument has been translated into most major languages including Arabic [12, 22]. The EuroQuol-5Dimensions (EQ-5D) has been described as a valid and reliable instrument to assess HRQoL in different populations [23-25].

The Arabic (Jordan) EQ-5D version was used to measure QoL among the elderly in this study [12]. In March 2013, permission to use the validated Arabic version was obtained from the EuroQoL group. The EQ-5D-5L consists of a descriptive system and the EQ visual analogue scale (EQ-VAS). The descriptive system includes five dimensions (mobility, self-care, usual activities, pain/discomfort, and anxiety/depression) and each dimension includes five levels of coding: $1=$ no problems; 2 = slight problems; $3=$ moderate problems; $4=$ severe problems; and $5=$ extreme problems. In addition, respondents reported self-rated health on an EQ-VAS with endpoints labeled 100 (the best health you can imagine) and 0 (the worst health you can imagine).

\section{Statistical analysis}

Data were analyzed using the Statistical Package for the Social Sciences (SPSS) package, version 20 (SPSS Inc., Chicago, IL). Descriptive statistics were used to characterize the sample according to PA and HRQoL variables. Kruskal-Wallis tests were performed on the ordinal variables of the PA groups' three levels to determine differences between the groups according to categorical variables such as gender, body mass index (BMI), and the prevalence of comorbid conditions. MannWhitney $U$ tests were performed on the ordinal variables of the EQ-5D. The independent sample $t$-test was performed on the EQ-VAS to determine differences between women and men and between the three physical activity groups (low, moderate, and high). Spearman's rank correlation coefficient was used to examine the correlation between EQ-5D and level of PA. Statistical significance was set at $\mathrm{P}<0.05$.

\section{Results}

The mean age of the participants was $68.15 \pm 6.74$ years (range: 60 - 91 years), and $76.6 \%$ of the participants lived with their families and $23.4 \%$ of the participants lived alone. About $42 \%$ of the participants had less than 6 years of education. The majority of the participants $(92 \%)$ were fully independent in the 
Table 2. Clinical Characteristics Among Different Physical Activity Groups

\begin{tabular}{|c|c|c|c|c|}
\hline Variables & Low PA group $(n=74)$ & Moderate PA group $(n=85)$ & High PA group $(n=17)$ & $\mathbf{P}$ \\
\hline Gender (female/male (n)) & $57 / 17$ & $52 / 33$ & $6 / 11$ & 0.001 \\
\hline BMI (kg/m²) (mean (SD)) & $32(5.1)$ & $30(4.9)$ & $29(5.0)$ & 0.029 \\
\hline Diagnosed disease (\%) & 97 & 81 & 65 & $<0.001$ \\
\hline Cardiovascular (\%) & 31 & 17 & 12 & 0.01 \\
\hline Diabetes $(\%)$ & 50 & 22 & 6 & $<0.001$ \\
\hline Hypertension (\%) & 64 & 44 & 12 & $<0.001$ \\
\hline Osteoporosis (\%) & 31 & 7 & 6 & $<0.001$ \\
\hline Hyperlipidemia (\%) & 24 & 13 & 6 & 0.072 \\
\hline
\end{tabular}

Values are presented as percentages, or mean (SD). SD: standard deviation; BMI: body mass index; n: number of cases.

Table 3. Health-Related Quality of Life (HRQoL) According to Gender

\begin{tabular}{|c|c|c|c|}
\hline HRQoL variables & Women $(n=115)$ & Men $(n=61)$ & $\mathbf{P}$ \\
\hline \multicolumn{4}{|l|}{ Mobility, n (\%) } \\
\hline I have no problems in walking about & $34(30)$ & $31(51)$ & \\
\hline I have slight problems in walking about & $59(51)$ & $19(31)$ & \\
\hline I have moderate problems in walking about & $15(13)$ & $9(15)$ & 0.030 \\
\hline I have severe problems in walking about & $7(6)$ & $2(3)$ & \\
\hline I am unable to walk about & $0(0)$ & $0(0)$ & \\
\hline \multicolumn{4}{|l|}{ Self-care, n (\%) } \\
\hline I have no problems washing or dressing myself & $65(57)$ & $37(61)$ & \\
\hline I have slight problems washing or dressing myself & $27(23)$ & $13(21)$ & \\
\hline I have moderate problems washing or dressing myself & $19(16)$ & $10(16)$ & 0.583 \\
\hline I have severe problems washing or dressing myself & $3(3)$ & $1(2)$ & \\
\hline I am unable to wash or dress myself & $1(1)$ & $0(0)$ & \\
\hline \multicolumn{4}{|c|}{ Usual activities (e.g. work, study, housework, family or leisure activities), n (\%) } \\
\hline I have no problems doing my usual activities & $37(32)$ & $18(29)$ & \\
\hline I have slight problems doing my usual activities & $32(28)$ & $20(33)$ & 0.850 \\
\hline I have moderate problems doing my usual activities & $36(31)$ & $15(25)$ & \\
\hline I have severe problems doing my usual activities & $7(6)$ & $8(13)$ & \\
\hline I am unable to do my usual activities & $3(3)$ & $0(0)$ & \\
\hline \multicolumn{4}{|l|}{ Pain/discomfort, n (\%) } \\
\hline I have no pain or discomfort & $18(15)$ & $17(28)$ & \\
\hline I have slight pain or discomfort & $55(48)$ & $28(46)$ & 0.057 \\
\hline I have moderate pain or discomfort & $30(26)$ & $11(18)$ & \\
\hline I have severe pain or discomfort & $10(9)$ & $5(8)$ & \\
\hline I have extreme pain or discomfort & $2(2)$ & $0(0)$ & \\
\hline \multicolumn{4}{|l|}{ Anxiety/depression, n (\%) } \\
\hline I am not anxious or depressed & $30(26)$ & $20(33)$ & \\
\hline I am slightly anxious or depressed & $52(45)$ & $29(48)$ & 0.108 \\
\hline I am moderately anxious or depressed & $14(12)$ & $10(16)$ & \\
\hline I am severely anxious or depressed & $18(16)$ & $2(3)$ & \\
\hline I am extremely anxious or depressed & $1(1)$ & $0(0.0)$ & \\
\hline EQ-VAS (mean (SD)) & $70.4(13.40)$ & $75.8(14.38)$ & 0.543 \\
\hline
\end{tabular}

SD: standard deviation; HRQoL: health-related quality of life; n: number of cases. 
Table 4. Health-Related Quality of Life (HRQoL) Measures Among Physical Activity (PA) Groups

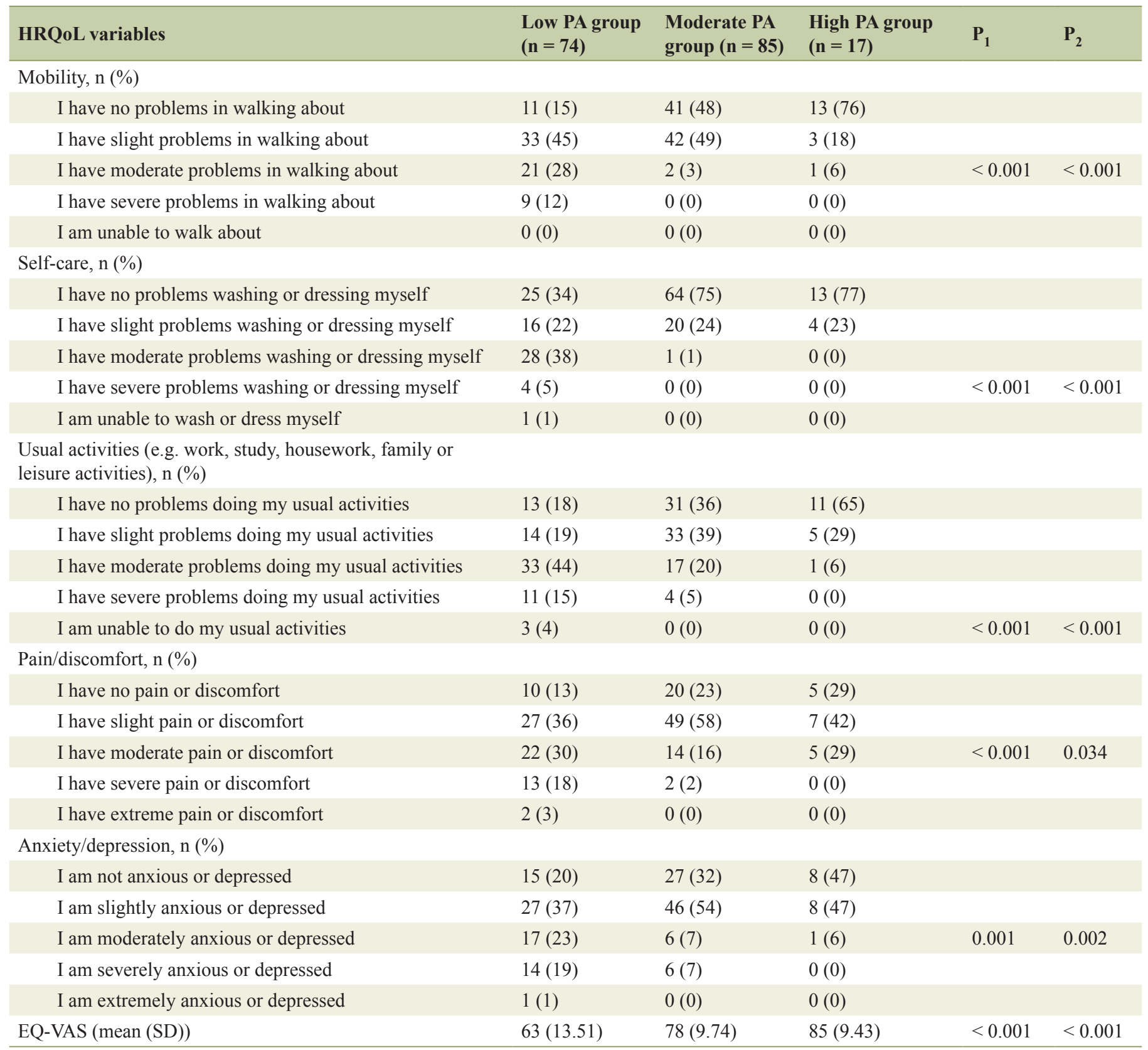

$\mathrm{P}_{1}$ : comparison between low and moderate PA groups; $\mathrm{P}_{2}$ : comparison between low and high PA groups.

BADL, and $8 \%$ were partially independent according to the Katz index. The majority of the participants $(86.4 \%)$ recorded a diagnosed disease such as hypertension (48.9\%), musculoskeletal (54.0\%), and cardiovascular disease (22.2\%). Clinical characteristics of the participants in relation to comorbidity are illustrated in Table 1 and Table 2.

Descriptive values of PA variables showed that $50 \%$ of the women and $28 \%$ of the men walked less than $150 \mathrm{~min} / \mathrm{week}$, which is also the definition of the low PA group in the present study (Table 1). In addition, $17 \%$ of the women and $79 \%$ of the men spent less than $150 \mathrm{~min} /$ week doing household activities,
$69 \%$ of the women and $6 \%$ of the men spent more than 300 $\mathrm{min} /$ week doing household activities, and $70 \%$ of the women and $46 \%$ of the men spent less than $150 \mathrm{~min} /$ week participating in outdoor activities.

Results of HRQoL measures showed that $64 \%$ of the participants recorded problems walking, ranging from slight problems to moderate and severe problems. In addition, $68.7 \%$ of the participants indicated that they had problems doing their usual activities. The majority of the participants (about $80.1 \%$ ) recorded having pain or discomfort ranging from slight to extreme pain. No significant differences were recorded according 
Table 5. Correlation Between HRQoL Variables and PA Level

\begin{tabular}{|llll}
\hline Variable & & \multicolumn{2}{c}{$\begin{array}{c}95 \% \text { confidence } \\
\text { interval }\end{array}$} \\
\cline { 3 - 4 } & & Lower & Upper \\
\hline $\begin{array}{l}\text { HRQoL variables } \\
\text { Mobility }\end{array}$ & -0.630 & -0.669 & -0.418 \\
Self-care & -0.526 & -0.614 & -0.352 \\
\hline Usual activities & -0.536 & -0.617 & -0.356 \\
\hline Pain/discomfort & -0.353 & -0.458 & -0.174 \\
\hline Anxiety/depression & -0.381 & -0.479 & 0.197 \\
\hline EQ visual analogue scale & 0.657 & 0.463 & 0.706 \\
\hline
\end{tabular}

$r_{s}$ : Spearman's correlation coefficient; HRQoL: health-related quality of life; EQ: Euro Quality.

to gender on all HRQoL dimensions except on the mobility dimension. Men recorded a mobility median score of 1 (I have no problem in walking about) and women recorded mobility median score of 2 (I have slight problems in walking about) (Table 3).

Significant differences between the three PA groups were recorded on the five dimensions of EU-QoL and EQ-VAS, where the higher PA groups recorded higher scores in all EUQoL dimensions (Table 4). Significant correlations were also recorded between the five dimensions of (EQ-5D-5L) and level of PA in terms of walking minutes $(\mathrm{P}<0.001)$ (Table 5).

\section{Discussion}

\section{Methodological consideration}

Determining a precise PA measurement to be used for elderly persons is very difficult due to physiological and cognitive changes that occur with aging [26]. This challenge can be more intense in a circumstance where the available valid measures for assessing PA in elderly $[20,21]$ cannot be entirely applied according to different domains of PA within diverse cultural contexts [1]. Therefore, this study uses an adapted questionnaire that considers social and cultural issues. The PA-SCAQ was constructed based on the WHO recommendations on PA, but it also considers some culturally applicable items of the valid PA measures for elderly persons [20, 21]. The prevalent domain of PA among the elderly Palestinians is more revolved around activities such as walking, gardening, and household chores [1]. Findings of the PA-SCAQ pilot testing corresponded with these more common activities. Thus we believe the PA-SCAQ had good face validity and enabled us to obtain the required descriptive statistics about PA domains among the elderly.

We used the data obtained from the PA-SCAQ to determine PA levels. The WHO [1] recommends that elderly persons should do at least $150 \mathrm{~min}$ of MIA-PA throughout the week. However, for additional health benefits, increasing MIA-PA to 300 min per week is recommended. The MIA-PA is defined as "activity in which the body's large muscles move in a rhythmic manner for a sustained period of time. Examples include walking, running, swimming, and bicycling" [1]. In our study, MIA-PA was typically obtained through walking, so levels of PA were determined using walking duration. Walking is one of the most common PAs among elderly persons and can easily be adapted into daily life [27]. Daily walks for at least 30 min have shown to be positively related to leg muscle strength and self-rated physical fitness among elderly [28].

The two major domains of interest for studying HRQoL in this study were functioning and subjective well-being [29]. To evaluate functioning, we used the EQ-5D-5L to determine mobility, self-care, and usual activities. To evaluate subjective well-being, we used the EQ-5D-5L to determine pain/discomfort, anxiety/depression, and VAS. Furthermore, the EQ-5D$5 \mathrm{~L}$ is a valid measure for Arabic speaking elderly [12]. EQ$5 \mathrm{D}-5 \mathrm{~L}$ was found to be a practical and simple tool for elderly populations $[23,24]$. Because about $42 \%$ of the participants in this study had less than 6 years of education, using EQ-5D-5L helped achieve a good response rate.

A possible limitation in this study was that the PA-SCAQ determines an overall description of PA level based on the walking minutes without quantifying various types of PA, an issue that was challenging to address within the scope of the present study.

\section{Results' discussion}

In this study, women constituted $77.0 \%$ of the low PA group and the majority of the participants $(86.4 \%)$ reported having at least one diagnosed disease. The low PA group recorded higher prevalence of chronic diseases such as cardiovascular, hypertension, and diabetes. Moderate intensity activities such as walking, gardening, or light sports appear to have beneficial effects and lead to a $30-50 \%$ reduction in cardiovascular disease in women [4]. Several studies have shown that regular moderate intensity of PA such as walking has significant benefits for health and for the treatment of a number of diseases [3, $5,6]$. Accordingly, increasing PA among elderly persons has become an international priority [1], so the elderly have been encouraged to regularly participate in moderate intensity activities $[6,9]$, culturally and age appropriate PA interventions for elderly are necessary to be established [1].

Higher PA groups recorded better values in all EQ-5D-5L dimensions. In the functioning domain, compared with the low PA group, the moderate and the high PA group recorded better scores on mobility $(\mathrm{P}<0.001)$. Women recorded lower scores on the mobility dimension of the HRQoL, and they constituted the majority of the low PA active group, findings that support the association between level of PA and HRQoL. This result might be attributed to the fact that more elderly women than elderly men are homebound. Because women are more likely to spend time performing household activities, their mobility level in relation to walking outdoors may be affected. Frandin et al found [28] that walkers had a more positive attitude towards PA as well as a higher estimation of their own physical fitness than non-walkers. 
The high PA group recorded higher values for self-care and the usual activities represented in the EQ-5D. Hence, selfcare and usual activities affect independence and a higher PA level contributes to reduced risk of disability in elderly [30]. Therefore, this study evaluated the association between PA levels and these components of the functioning domain of EQ$5 \mathrm{D}$.

We addressed the subjective well-being domain of HRQoL using data about pain/discomfort and anxiety/depression as well as reported self-rated health on the EQ-VAS. The state of well-being refers to an individual's feelings at the time they are expressed [31]. Almost half of the participants indicated having slight pain or discomfort and were slightly anxious or depressed. Significant differences were recorded on the variables of the subjective well-being domain with a significantly higher VAS score compared to the lower PA group. These findings are consistent with similar studies that have shown the protective effect of PA on depression and bodily pain among elderly $[3,32]$.

\section{Conclusion}

There were strong associations between higher levels of PA and all dimensions of HRQoL. The high PA group showed better values in all dimensions of HRQoL. In addition, the prevalence of comorbid conditions was higher in the low PA group. Therefore, adopting a physically active lifestyle may contribute to better health and HRQoL among the elderly.

\section{Conflict of Interest}

The authors report no conflict of interests.

\section{References}

1. Global Recommendations on Physical Activity for Health. Geneva, 2010.

2. Vagetti GC, Barbosa Filho VC, Moreira NB, Oliveira V, Mazzardo O, Campos W. Association between physical activity and quality of life in the elderly: a systematic review, 2000-2012. Rev Bras Psiquiatr. 2014;36(1):76-88.

3. Acree LS, Longfors J, Fjeldstad AS, Fjeldstad C, Schank $\mathrm{B}$, Nickel KJ, Montgomery PS, et al. Physical activity is related to quality of life in older adults. Health Qual Life Outcomes. 2006;4:37.

4. Beitz R, Doren M. Physical activity and postmenopausal health. J Br Menopause Soc. 2004;10(2):70-74.

5. Brach JS, Simonsick EM, Kritchevsky S, Yaffe K, Newman AB. The association between physical function and lifestyle activity and exercise in the health, aging and body composition study. J Am Geriatr Soc. 2004;52(4):502509.

6. Pedersen BK, Saltin B. Evidence for prescribing exercise as therapy in chronic disease. Scand J Med Sci Sports. 2006;16(Suppl 1):3-63.

7. Paterson DH, Jones GR, Rice CL. Ageing and physical activity: evidence to develop exercise recommendations for older adults. Can J Public Health. 2007;98(Suppl 2):S69-108.

8. Aoyagi Y, Shephard RJ. Habitual physical activity and health in the elderly: the Nakanojo Study. Geriatr Gerontol Int. 2010;10(Suppl 1):S236-243.

9. Svantesson U, Jones J, Wolbert K, Alricsson M. Impact of Physical Activity on the Self-Perceived Quality of Life in Non-Frail Older Adults. J Clin Med Res. 2015;7(8):585593.

10. The Development of the World Health Organization Quality of Life Assessment Instrument (the WHOQOL). In: Orley J, Kuyken W, editors. Quality of Life Assessment: International Perspectives. Springer Berlin Heidelberg; 1994; p. 41-57.

11. Coons SJ, Rao S, Keininger DL, Hays RD. A comparative review of generic quality-of-life instruments. Pharmacoeconomics. 2000;17(1):13-35.

12. Aburuz S, Bulatova N, Twalbeh M, Gazawi M. The validity and reliability of the Arabic version of the EQ-5D: a study from Jordan. Ann Saudi Med. 2009;29(4):304-308.

13. Pattanaphesaj J, Thavorncharoensap M. Measurement properties of the EQ-5D-5L compared to EQ-5D-3L in the Thai diabetes patients. Health Qual Life Outcomes. 2015;13:14.

14. Ortlieb S, Gorzelniak L, Nowak D, Strobl R, Grill E, Thorand B, et al. Associations between Multiple Accelerometry-Assessed Physical Activity Parameters and Selected Health Outcomes in Elderly People-Results from the KORA-Age Study. 2014.

15. van Oostrom SH, Smit HA, Wendel-Vos GC, Visser M, Verschuren WM, Picavet HS. Adopting an active lifestyle during adulthood and health-related quality of life: the Doetinchem Cohort Study. Am J Public Health. 2012;102(11):e62-68.

16. Balboa-Castillo T, Leon-Munoz LM, Graciani A, Rodriguez-Artalejo F, Guallar-Castillon P. Longitudinal association of physical activity and sedentary behavior during leisure time with health-related quality of life in community-dwelling older adults. Health Qual Life Outcomes. 2011;9:47.

17. Choi M, Prieto-Merino D, Dale C, Nuesch E, Amuzu A, Bowling A, Ebrahim S, et al. Effect of changes in moderate or vigorous physical activity on changes in healthrelated quality of life of elderly British women over seven years. Qual Life Res. 2013;22(8):2011-2020.

18. Katz S, Ford AB, Moskowitz RW, Jackson BA, Jaffe MW. Studies of Illness in the Aged. The Index of Adl: A Standardized Measure of Biological and Psychosocial Function. JAMA. 1963;185:914-919.

19. Brorsson B, Asberg KH. Katz index of independence in ADL. Reliability and validity in short-term care. Scand J Rehabil Med. 1984;16(3):125-132.

20. Grimby G. Physical activity and muscle training in the elderly. Acta Med Scand Suppl. 1986;711:233-237.

21. Washburn RA, Smith KW, Jette AM, Janney CA. The Physical Activity Scale for the Elderly (PASE): development and evaluation. J Clin Epidemiol. 1993;46(2):153162. 
22. EuroQol--a new facility for the measurement of healthrelated quality of life. Health Policy. 1990;16(3):199-208.

23. Holland R, Smith RD, Harvey I, Swift L, Lenaghan E. Assessing quality of life in the elderly: a direct comparison of the EQ-5D and AQoL. Health Econ. 2004;13(8):793805.

24. Tidermark J, Bergstrom G. Responsiveness of the EuroQol (EQ-5D) and the Nottingham Health Profile (NHP) in elderly patients with femoral neck fractures. Qual Life Res. 2007;16(2):321-330.

25. Obradovic M, Lal A, Liedgens H. Validity and responsiveness of EuroQol-5 dimension (EQ-5D) versus Short Form-6 dimension (SF-6D) questionnaire in chronic pain. Health Qual Life Outcomes. 2013;11:110.

26. Kowalski K, Rhodes R, Naylor PJ, Tuokko H, MacDonald $\mathrm{S}$. Direct and indirect measurement of physical activity in older adults: a systematic review of the literature. Int J Behav Nutr Phys Act. 2012;9:148.

27. Atalay OT, Cavlak U. The impact of unsupervised regular walking on health: A sample of Turkish middle-aged and older adults. European Review of Aging and Physical Activity. 2012;9:71-79.

28. Frandin K, Grimby G, Mellstrom D, Svanborg A. Walking habits and health-related factors in a 70-year-old population. Gerontology. 1991;37(5):281-288

29. Stewart AL, King AC. Evaluating the efficacy of physical activity for influencing quality-of-life outcomes in older adults. Annals of Behavioral Medicine. 1991;13:108-116.

30. Tak E, Kuiper R, Chorus A, Hopman-Rock M. Prevention of onset and progression of basic ADL disability by physical activity in community dwelling older adults: a meta-analysis. Ageing Res Rev. 2013;12(1):329-338.

31. Kunzmann U, Little TD, Smith J. Is age-related stability of subjective well-being a paradox? Cross-sectional and longitudinal evidence from the Berlin Aging Study. Psychol Aging. 2000;15(3):511-526.

32. Strawbridge WJ, Deleger S, Roberts RE, Kaplan GA. Physical activity reduces the risk of subsequent depression for older adults. Am J Epidemiol. 2002;156(4):328334. 